\title{
A Segmentation Model Combining Local and Global Energy Based on Local Entropy
}

\author{
Zhang Ying $^{1}$ and Paul Yanne ${ }^{2 *}$ \\ ${ }^{1}$ College of Mechanical and Electronic Engineering, Northwest A\&F University \\ ${ }^{2}$ College of Information Engineering, Northwest A\& $F$ University \\ ${ }^{1}$ windflycq@foxmail.com, ${ }^{2}$ pyanne@nwsuaf.edu.cn
}

\begin{abstract}
Image segmentation based partial differential equation is very popular in image analysis and computer vision. This article presents a geometric active contour model based on local entropy for segmentation. In the proposed method, energy functional item of this model contains local energy and global energy items, and uses local entropy of each point on the contour line to determine the proportion of each kind of energy. The combined method allows the contour for the rapid evolution and convergence to the final edge after iterations. Experiment results on both synthetic and real images demonstrate the efficiency and accuracy of this model.
\end{abstract}

Keywords: image segmentation, partial differential, $C$-V model, local entropy

\section{Introduction}

Image segmentation is an important issue in computer vision and image processing. The purpose of image segmentation is to separate and extract interesting regions and then to divide them into different areas according to the characteristics. Image segmentation result is the basic foundation for further image analysis and processing. Due to the complexity of the image being segmented, there is no universal image segmentation method. Among all image segmentation methods, the one based on partial differential equations has become a hot research topic because of its solid mathematical theory support.

Active contour model (ACM) [1] is one of the most successful methods of image segmentation based on partial differential equations. ACMs can be categorized into two types: parametric active contour models (PACM) [2-5] and the geometric active contour models (GACM) [6-11].

GACMs have many advantages over PACMs [12]. First, region-based models utilize the statistical information inside and outside the contour to control the evolution, which is less sensitive to noise and has better performance for images with weak edges or without edges. Second, they are significantly less sensitive to the location of the initial contour and then can efficiently detect the exterior and interior boundaries simultaneously. One of the most popular region-based models is the $\mathrm{C}-\mathrm{V}$ model [13], which is based on Mumford-Shah segmentation techniques and has been successfully applied to binary phase segmentation [14].

Among the GACMs, using the gray statistics of image homogeneous regions, $\mathrm{C}-\mathrm{V}$ model is based on global information and hence ineffective for images with the uneven distribution of gray scale; Only considering local gray information, the LBF model is more sensitive and susceptible to noise [15], its segmentation results may lead to oversegmentation or wrong segmentation. This paper presents a segmentation model

${ }^{*}$ Corresponding Author 
combining local and global energy based on local entropy. It combines the advantages of the global properties of $\mathrm{C}-\mathrm{V}$ model and the local properties of LBF model.

\section{Background}

\subsection{The $\mathbf{C}-\mathbf{V}$ Model}

C-V model proposed by T.F.Chan and L.A.Vese can be seen as a special case of the Munford-Shah model. Let image $I(x, y)$ is a function in the domain $\Omega, C$ is a closed curve, $C_{0}$ represents the target profile curve, this curve is divided image $I$ into two regions: an inner region inside $(C)$ and an outer region outside $(C)$, its average gray is represented by $c_{1}$ and $c_{2}$. The $\mathrm{C}-\mathrm{V}$ model proposed the following energy functional:

$$
F(C)=\int_{\text {inside }(C)}\left|I-c_{1}\right|^{2} d x d y+\int_{\text {outside }(C)}\left|I-c_{2}\right|^{2} d x d y
$$

In the above formula, when the evolution of the curve located at the boundary position, that is $C=C_{0}$, its energy functional minimum. Plus curve length term, energy functional of $\mathrm{C}-\mathrm{V}$ model as follow:

$E\left(C, c_{1}, c_{2}\right)=\lambda_{1} \iint_{\text {inside }(C)}\left|I-c_{1}\right|^{2} d x d y+\lambda_{2} \iint_{\text {outside }(C)}\left|I-c_{2}\right|^{2} d x d y+\mu$ length $(C)$

where $\lambda_{1}, \lambda_{2}>0$ are fixed parameters. $H_{\varepsilon}(z)$ represents Heaviside operator and $\delta_{\varepsilon}(\phi)$ represents Dirac operator. Minimizing the above energy functional by using the steepest descent method, we obtain the level set variational equations:

$$
\frac{\partial \phi}{\partial t}=\delta_{\varepsilon}(\phi)\left\lceil\mu \nabla \cdot \frac{\nabla \phi}{|\nabla \phi|}-\lambda_{1}\left(I(x, y)-c_{1}\right)^{2}+\lambda_{2}\left(I(x, y)-c_{2}\right)^{2}\right]
$$

Where $c_{1}$ and $c_{2}$ are represented as follow:

$$
\left\{\begin{array}{l}
c_{1}=\frac{\int_{\Omega} I(x, y) H(\phi(x, y)) d x d y}{\int_{\Omega} H(\phi(x, y)) d x d y} \\
c_{2}=\frac{\int_{\Omega} I(x, y)(1-H(\phi(x, y))) d x d y}{\int_{\Omega}(1-H(\phi(x, y))) d x d y}
\end{array}\right.
$$

Obviously, in Eq. (4), $c_{1}$ and $c_{2}$ represent the average gray of inside and outside the contour. They are represented the global properties of the image $I . \mathrm{C}-\mathrm{V}$ model is based on global information and hence ineffective for images with the uneven distribution of gray scale.

\subsection{The LBF Model}

Li Chunming et al., proposed the LBF model. This model introduces a kernel function to define a local binary fitting energy in a variational formulation therefore is able to segment images with intensity inhomogeneity.

Let image $I(x, y)$ is a function in the domain $\Omega, C$ is a contour in the image. Local fitting energy functional of each point $x \in \Omega$ as follow: 


$$
\begin{aligned}
E^{L B F}\left(C, f_{1}, f_{2}\right) & =\lambda_{1} \int_{\Omega} \int_{\text {inside }(C)} K_{\sigma}(x-y)\left|I(y)-f_{1}(x)\right|^{2} d x d y \\
& +\lambda_{2} \int_{\Omega} \int_{\text {outside }(C)} K_{\sigma}(x-y)\left|I(y)-f_{2}(x)\right|^{2} d x d y
\end{aligned}
$$

Where $\lambda_{1}, \lambda_{2}>0$ are fixed parameters. $K_{\sigma}(x, y)$ is a Gaussian kernel function, $\sigma$ is the standard deviation. $x$ is the center point, and points $y$ near point $x . f_{1}(x)$ and $f_{2}(x)$ are two numbers that fit image intensities near point $x$, when the evolution of the curve located at the boundary position, its energy functional minimum. $f_{1}(x)$ and $f_{2}(x)$ can be rewritten as:

$$
\left\{\begin{array}{c}
f_{1}(x)=\frac{K_{\sigma} *\left[H_{\varepsilon}(\phi) I(x)\right]}{K_{\sigma} * H_{\varepsilon}(\phi)} \\
f_{2}(x)=\frac{K_{\sigma} *\left[\left(1-H_{\varepsilon}(\phi)\right) I(x)\right]}{K_{\sigma} *\left(1-H_{\varepsilon}(\phi)\right)}
\end{array}\right.
$$

Add the distance regularizing term and the length of the zero level curve, the entire energy functional can be written as:

$E_{\text {total }}^{L B F}=E^{L B F}\left(C, f_{1}, f_{2}\right)+\mu \int_{\Omega} \frac{1}{2}(|\nabla \phi|-1)^{2} d x+v \int_{\Omega} \delta(\phi(x, y))|\nabla \phi(x, y)| d x$

Minimizing the above energy functional with gradient descent flow, we get the level set function equation:

$\frac{\partial \phi}{\partial t}=\mu\left(\nabla^{2} \phi-\operatorname{div}\left(\frac{\nabla \phi}{|\nabla \phi|}\right)\right)+v \delta_{\varepsilon}(\phi) d i v\left(\frac{\nabla \phi}{|\nabla \phi|}\right)-\delta_{\varepsilon}(\phi)\left(\lambda_{1} \mathrm{e}_{1}-\lambda_{2} \mathrm{e}_{2}\right)$

When $\lambda_{1}=\lambda_{2}, e_{1}$ and $e_{2}$ are the functions as follow:

$\left\{\begin{array}{l}e_{1}(x)=\int_{\Omega} K_{\sigma}(y-x)\left|I(x)-f_{1}(y)\right|^{2} d y \\ e_{2}(x)=\int_{\Omega} K_{\sigma}(y-x)\left|I(x)-f_{2}(y)\right|^{2} d y\end{array}\right.$

The calculation of $f_{1}(x)$ and $f_{2}(x)$ is relatively simple, unlike other models of complex partial differential equations to be solved, and therefore the value LBF model calculations faster. In addition, this model has a certain degree of noise immunity, and can reduce the redundancy profile generation.

\section{The Proposed Model}

\subsection{Local Entropy Parameter}

In information theory, entropy is a measure of the uncertainty of the probability of an event, it can effectively reflect the information contained in the event [16]. Local entropy with image size of $M \times N$ is defined as follows:

$E=-\sum_{i=0}^{L-1} P_{i} \log P_{i}$

Where $P_{i}=n /(M \times N), P_{i}$ is the probability of gray level $i$ that appears in the 
image, $L$ is a maximum gray level, $n_{i}$ is the number of gray levels pixels having a gray level of $i$.

Let the pixel $r$ on the curve $C$ as the center of a neighborhood with a window size of $M_{k} \times N_{k}$, then $E_{r} E_{k}$ is the image local entropy. Local entropy can describe the distribution of the degree of dispersion and noise of each gray level in an image.

In this paper, we calculate local entropy of $5 \times 5$ pixels in size near the contour lines and defined the following formula:

$\gamma=E_{r} / \mathrm{max} E_{r}$

As can be seen from the above equation, $0<\gamma \leq 1$, When $r$ is in the gray uniform region, $E_{r}$ is greater, so $\gamma$ close to 1 ; on the contrary, $r$ is in the uneven gradation or noise area, $E_{r}$ is smaller.

\subsection{Energy Functional}

$\mathrm{C}-\mathrm{V}$ model based on global information, the problem of this model is the uneven distribution of gray scale image segmentation ineffective; LBF model is more sensitive and susceptible to noise, and results may lead to over-segmentation or split a bad situation. This paper presents a LLG model. This model based on local entropy and it combines the advantages of global properties of C-V model and local properties model of LBF model.

The LLG model combining local and global energy, the energy functional we proposed as follows:

$\varepsilon^{L L G}=E^{L}\left(C, f_{1}, f_{2}\right)+E^{G}\left(C, c_{1}, c_{2}\right)$

The first item in the formula represents the local energy term, which is expressed as follows:

$E^{L}\left(C, f_{1}(x), f_{2}(x)\right)=\gamma \sum_{i=1}^{2} \lambda_{i} \int_{\Omega_{i}} K(x-y)\left|I(y)-f_{i}(x)\right|^{2} d y$

The second global energy in the model is based on the energy functional:

$E^{G}\left(C, c_{1}, c_{2}\right)=(1-\gamma) \sum_{i=1}^{2} \lambda_{i} \iint_{\Omega_{i}}\left|I-c_{i}\right|^{2} d x d y$

Then, the entire energy functional can be written in the following form:

$$
\begin{aligned}
\varepsilon^{L L G}= & (1-\gamma)\left(\left(\lambda_{1} \int_{\Omega} \int_{\text {inside }(C)} K_{\sigma}(x-y)\left|I(y)-f_{1}(x)\right|^{2} d x d y+\right.\right. \\
& \left.+\lambda_{2} \int_{\Omega} \int_{\text {outside }(C)} K_{\sigma}(x-y)\left|I(y)-f_{2}(x)\right|^{2} d x d y\right) \\
& +\gamma\left(\lambda_{3} \iint_{\text {inside }(C)}\left|I-c_{1}\right|^{2} d x d y+\lambda_{4} \iint_{\text {outside }(C)}\left|I-c_{2}\right|^{2} d x d y\right)
\end{aligned}
$$

Where $0<\gamma \leq 1$, and it is used to adjust the local energy and the global energy in the model. When the profile curve located gray uniform area, $\gamma$ value is the maximum value and the global energy accounted for the largest proportion, otherwise the global energy accounted for the less proportion.

In this model, in order to reduce the computational complexity, adding a new sign distance function:

$\varepsilon(C)=\varepsilon^{L L G}+$ vlength $(C)$ 
Approximate so, it is used to ensure that the curve evolution approximate signed distance function, thereby reducing the running time of the algorithm.

$R_{p}(\phi)=\int_{\Omega} p(|\nabla \phi|) d x$

The total LLG model energy functional can be written as follows:

$\varepsilon(C)=\varepsilon^{L L G}+$ vlength $(C)+R_{p}(\phi)$

Corresponding gradient descent flow obtained from equation (18) as the following gradient flow:

$$
\begin{aligned}
\frac{\partial \phi}{\partial t} & \left.=(1-\gamma) \times \delta_{\varepsilon}(\phi) \mid \mu \nabla \cdot \frac{\nabla \phi}{|\nabla \phi|}-\lambda_{1}\left(I(x, y)-c_{1}\right)^{2}+\lambda_{2}\left(I(x, y)-c_{2}\right)^{2}\right] \\
& -\gamma \times \delta_{\varepsilon}(\phi)\left(\lambda_{3} \mathrm{e}_{1}-\lambda_{4} \mathrm{e}_{2}\right)+\mu \operatorname{div}\left(d_{p}(|\nabla \phi|) \nabla \phi\right)+v \delta_{\varepsilon}(\phi) \operatorname{div}\left(\frac{\nabla \phi}{|\nabla \phi|}\right)
\end{aligned}
$$

We use the finite difference method to solve this equation as follow:

$$
\begin{aligned}
& \operatorname{div}\left(\frac{\nabla \phi}{|\nabla \phi|}\right)=\frac{\phi_{i+1, j+1}^{n+1}+\phi_{i-1, j+1}^{n+1}+\phi_{i+1, j-1}^{n+1}+\phi_{i+1, j+1}^{n+1}-4 \phi_{i, j}^{n+1}}{h^{2}} \\
& |\nabla \phi|=\left(\frac{\partial^{2} \phi}{\partial x^{2}}+\frac{\partial^{2} \phi}{\partial y^{2}}\right)^{\frac{1}{2}}
\end{aligned}
$$

\section{Implementation}

The temporal partial derivative $\partial \phi / \partial t$ in (19) is approximated by the forward difference:

$\frac{\partial \phi}{\partial t}=\frac{\phi_{i, j}^{n+1}-\phi_{i, j}^{n}}{t}$

Spatial partial derivatives $\partial \phi / \partial x$ and $\partial \phi / \partial y$ are approximated by the central difference as follow:

$$
\begin{aligned}
& \frac{\partial \phi}{\partial x}=\frac{\phi_{i+1, j}^{n}-\phi_{i-1, j}^{n}}{2 h} \\
& \frac{\partial \phi}{\partial y}=\frac{\phi_{i+1, j}^{n}-\phi_{i-1, j}^{n}}{2 h} \\
& \frac{\partial^{2} \phi}{\partial x^{2}}=\frac{\phi_{i+1, j}^{n}+\phi_{i-1, j}^{n}-2 \phi_{i, j}^{n}}{h^{2}} \\
& \frac{\partial^{2} \phi}{\partial y^{2}}=\frac{\phi_{i, j+1}^{n}+\phi_{i, j-1}^{n}-2 \phi_{i, j}^{n}}{h^{2}}
\end{aligned}
$$

In practice, the Dirac function $\delta_{\varepsilon}(x)$ in (19) is defined as the following function:

$$
\delta_{\varepsilon}(x)=H_{\varepsilon}^{\prime}(x)=\frac{1}{\pi} \frac{\varepsilon}{\varepsilon^{2}+x^{2}}
$$

We use the regularized $\operatorname{Dirac} \delta_{\varepsilon}(x)$ with $\varepsilon=1$ for segmentation in the paper. 
Algorithm steps of this model are as follows:

Step1: read the image, set an initial contour curve and the parameters;

Step2: initialize the level set function;

Step3: calculate local entropy at each point of the contour curve and substituted into the formula (11);

Step4: according to equation (18) the evolution of the energy equation;

Step5: by the formula (19) is the level set function iteration;

Step6: substituting the criterion to determine whether to meet the iteration stop condition. The equation (21) converges to stop evolution; otherwise Step 3.

\section{Experimental Results}

The proposed LLG method has been applied to a variety of synthetic and real images. The results show good performance of this method in various images.

For example, Figure 1 shows the result on a 95*95 pixel of synthetic image. Set the parameters $\Delta t=1, h=1, \lambda_{1}=1, \lambda_{2}=1, \sigma=3, \varepsilon=1, v=0.001 * 255 * 255$. In order to verify it is not sensitive to the location of initial contours, set initial contour at different positions, as shown in (a). Figure (b) shows the evolution of the contour. Figure (c), shows the segmentation results. The first one takes 10 iterations and the second one takes 50 iterations. This results show that the proposed method is robust to initialization and less sensitive to noises.

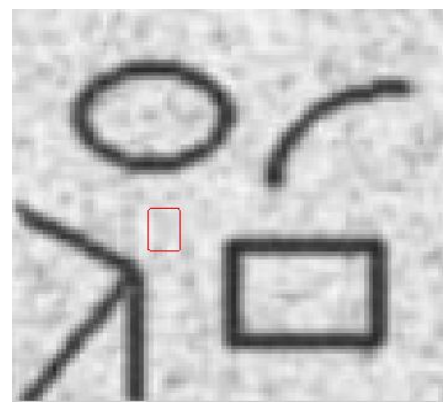

(a1)

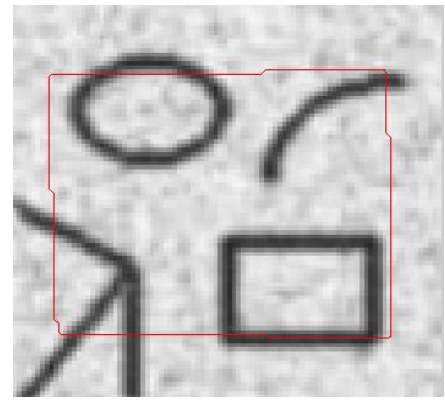

(a2)

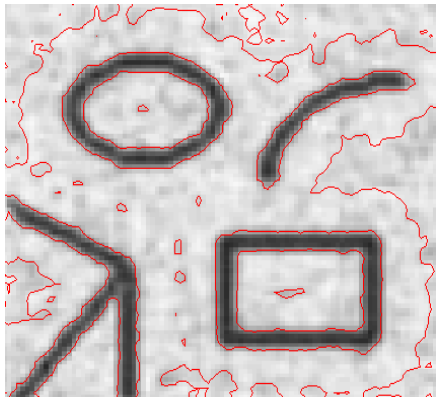

(b1)

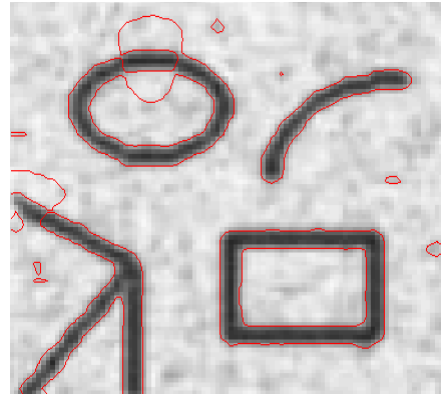

(b2)

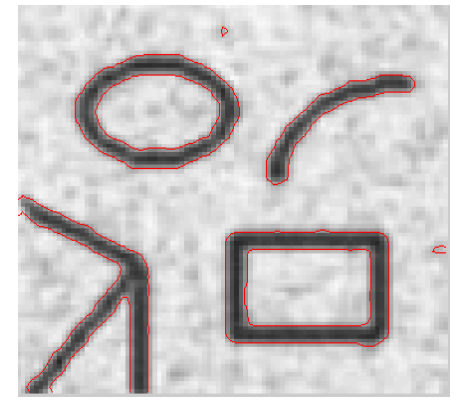

(c1)

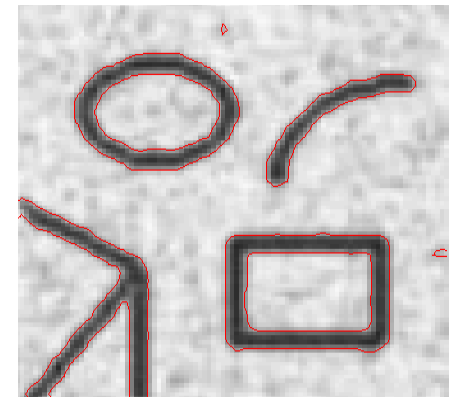

(c2)

(a) Initial Contour

(b) Evolution of the Contour

(c) Segmentation Results

The C-V model, LBF model and LLG method have been applied to uneven gray scale image, the results as showed in Figure 2. Set the parameters $\Delta t=1, h=1, \lambda_{1}=1$, $\lambda_{2}=1, \sigma=3, \varepsilon=1, v=0.001 * 255 * 255$. As can be seen from the figure, for uneven gray image segmentation the LLG model results are better than the other two models segmentation. 


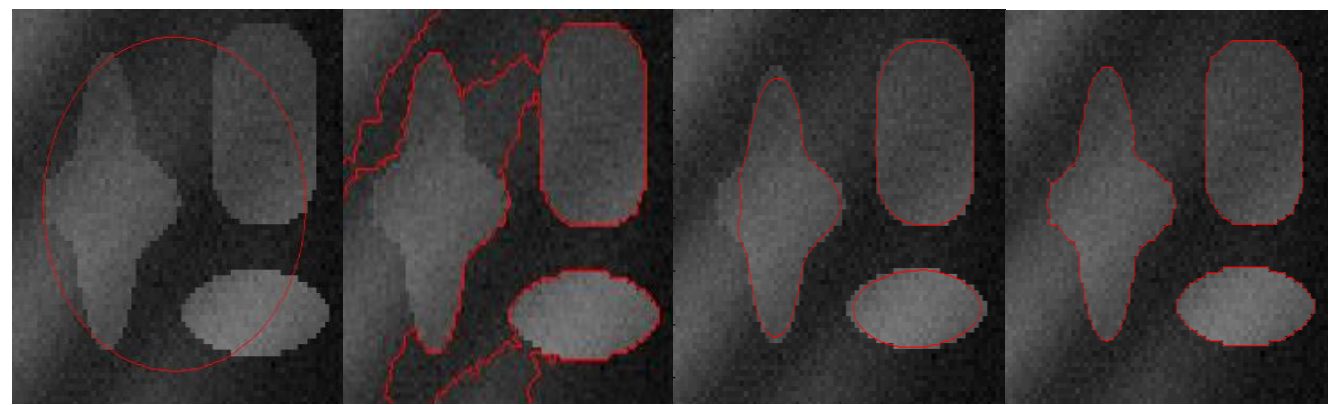

(a1)

(b1)

(c1)

(d1)

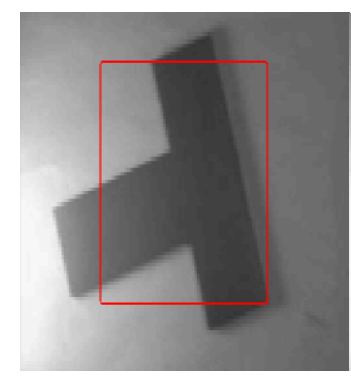

(a2)

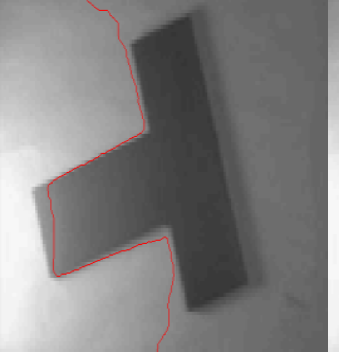

(b2)

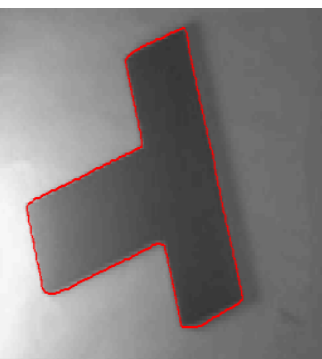

(c2)

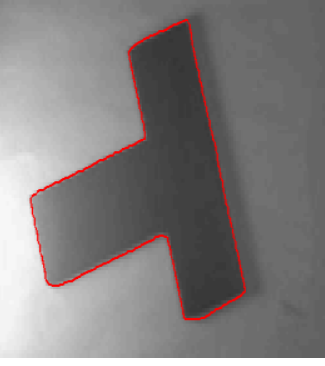

(d2)

(a) Initial Contour

(b) C-V Model

(c) LBF Model

(d) LLG Model

Figure 2. Uneven Gray Images Segmentation

Quantitative evaluation of image segmentation for three models as showed in Table 1, Error number of pixels of segmentation divided by the target object pixels, we get the segmentation error rate. Then we calculated the accuracy of segmentation.

Table 1. Quantitative Evaluation of Image Segmentation for Three Models

\begin{tabular}{|l|l|l|l|l|}
\hline Image & Size & C-V model & LBF model & LLG model \\
\hline Synthetic image & $79 \times 75$ & $56.3 \%$ & $88.1 \%$ & $99.6 \%$ \\
\hline Real image & $127 \times 96$ & $45.6 \%$ & $99.8 \%$ & $99.8 \%$ \\
\hline
\end{tabular}

Image segmentation results of the above comparison, experiments show that the GLBF model not only has the advantage of $\mathrm{C}-\mathrm{V}$ global energy model also has the advantage of local energy LBF model, broader scope, segmentation efficiency is higher.

\section{Conclusion}

In this paper, we present a new segmentation model based on local entropy. This model combines the advantages of global properties of $\mathrm{C}-\mathrm{V}$ model and local properties model of LBF model. We use local entropy to adjust the local energy and the global energy in the energy functional. When the profile curve located gray uniform area, the local entropy value is the maximum value and the local energy accounted for the largest proportion, otherwise the local energy accounted for the less proportion. This model allows for more flexible applications. The experimental results show that the model can well segment images with uneven gray distribution and noises, and is not sensitive to the initial outline. We propose LLG model, which is not only computationally more efficient than traditional segmentation model, but also allows for more flexible applications. 


\section{References}

[1] M. Kass, A. Witkin and D. Terzopoulos, "Snakes: active contour models", International Journal of Computer Vision, vol. 2, no. 1, (1998), pp. 321-331.

[2] P. Moallem, H. Tahvilian and S. A. Monadjemi, "Parametric active contour model using Gabor balloon energy for texture segmentation", Image and Video Processing, vol. 1, no. 14, (2015), pp. 1-8.

[3] N. Ray and S. Acton, "Motion gradient vector flow: an external force for tracking rolling leukocytes with shape and size constrained active contours", IEEE Transactions on Medical Imaging, vol. 23, no. 12, (2004), pp. 1466-1478.

[4] A. Tayyebia, B. C. Pijanowskib, M. Lindermanc and C. Grattona, "Comparing three global parametric and local non-parametric models to simulate land use change in diverse areas of the world ".Environmental Modelling \& Software, vol. 9, no. 59, (2014), pp. 202-221.

[5] B. Li and S. Acton, "Active contour external force using vector field convolution for image segmentation”. IEEE Transactions on Image Processing, vol. 16, no. 8, (2007), pp. 2096-2106.

[6] V. Caselles, R. Kimmelnd and G. Sapiro, "Geodesic active contours", International Journal of Computer Vision, vol. 22, no. 1, (1997), pp. 61-79.

[7] N. Krishnan and S. N. N. Sujatha, "A fast geometric active contour model with automatic region grid". Computational Intelligence and Computing Research (ICCIC), (2010), pp. 1- 8.

[8] G. Papandreou and P. Maragos, "Multigrid Geometric Active Contour Models", Image Processing, vol. 16, no. 1, (2007), pp. 229-240.

[9] C. Li, C. Kao, J. Gore and Z. Ding, "Minimization of region-scalable fitting energy for image segmentation”, IEEE Transactions on Image Processing, vol. 17, no. 10, (2008), pp. 1940-1949.

[10] X. Xiea and M. Mirmehdi, "MAC: Magnetostatic active contour model", IEEE Transactions on Pattern Analysis and Machine Intelligence, vol. 30, no. 4, (2008), pp. 632-646.

[11] K. Zhang, L. Zhang, H. Song and W. Zhou, "Active contours with selective local or global segmentation: a new formulation and level set method", Image and Vision Computing, vol. 28, no. 4, (2010), pp. 668-676.

[12] X. H. WANG and L. L. FANG, "Survey of Image Segmentation Based on Active Contour Model", Pattern Recognition and Artificial Intelligence, vol. 26, no. 8, (2013), pp.751-758.

[13] T. F. Chan and L. A. Vese, "Active Contours without Edges", IEEE Transactions on Image Processing, vol. 38, (2001), pp. 266-277.

[14] D. Mumford and J. Shah, "Optimal Approximations by Piecewise Smooth Functions and Associated Variational Problems", Communications on Pure and Applied Mathematics, vol. 42, no. 2, (1989), pp. 577-685.

[15] L. Chunming, K. Chiuyen and J. Gore, "Implicit active contours driven by local binary fitting energy", IEEE Conference on Computer Vision and Pattern Recognition (CVPR), Minneapolis, (2007), pp.1-7.

[16] C. E. Shannon, "A mathematical theory of communication", Bell System Technical Journal, vol. 5, no. 1, (2001), pp. 33-55.

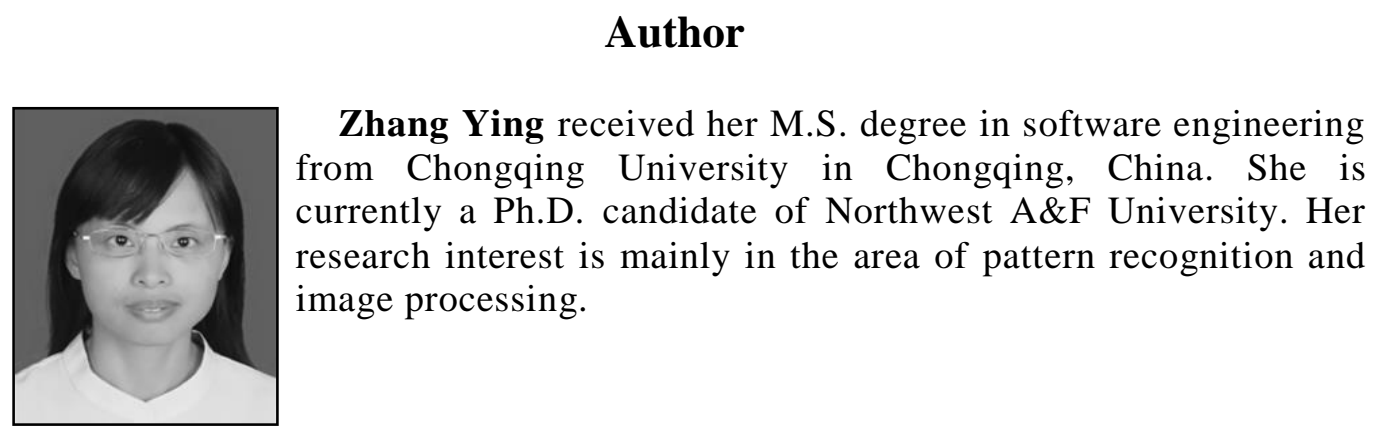

